\title{
A NOTE ON THE SPACES OF VARIABLE INTEGRABILITY AND SUMMABILITY OF ALMEIDA AND HÄSTÖ
}

\author{
HENNING KEMPKA AND JAN VYBÍRAL
}

(Communicated by Thomas Schlumprecht)

\begin{abstract}
We address an open problem posed recently by Almeida and Hästö. They defined the spaces $\ell_{q(\cdot)}\left(L_{p(\cdot)}\right)$ of variable integrability and summability and showed that $\| \cdot\left|\ell_{q(\cdot)}\left(L_{p(\cdot)}\right)\right| \mid$ is a norm if $q \geq 1$ is constant almost everywhere or if $1 / p(x)+1 / q(x) \leq 1$ for almost every $x \in \mathbb{R}^{n}$. Nevertheless, the natural conjecture (expressed also by Almeida and Hästö) is that the expression is a norm if $p(x), q(x) \geq 1$ almost everywhere. We show that $\left\|\cdot \mid \ell_{q(\cdot)}\left(L_{p(\cdot)}\right)\right\|$ is a norm if $1 \leq q(x) \leq p(x)$ for almost every $x \in \mathbb{R}^{n}$. Furthermore, we construct an example of $p(x)$ and $q(x)$ with $\min (p(x), q(x)) \geq 1$ for every $x \in \mathbb{R}^{n}$ such that the triangle inequality does not hold for $\left\|\cdot \mid \ell_{q(\cdot)}\left(L_{p(\cdot)}\right)\right\|$.
\end{abstract}

\section{INTRODUCTION}

For the definition of the spaces $\ell_{q(\cdot)}\left(L_{p(\cdot)}\right)$ we closely follow [1]. Spaces of variable integrability $L_{p(\cdot)}$ and variable sequence spaces $\ell_{q(\cdot)}$ were first considered in 1931 by Orlicz [5], but the modern development started with the paper 4]. We refer to [3] for an excellent overview of the vastly growing literature on the subject.

First of all we recall the definition of the variable Lebesgue spaces $L_{p(\cdot)}(\Omega)$, where $\Omega$ is a measurable subset of $\mathbb{R}^{n}$. A measurable function $p: \Omega \rightarrow(0, \infty]$ is called a variable exponent function if it is bounded away from zero. For a set $A \subset \Omega$ we denote $p_{A}^{+}=\operatorname{ess-sup}_{x \in A} p(x)$ and $p_{A}^{-}=\operatorname{ess-inf}_{x \in A} p(x)$; we use the abbreviations $p^{+}=p_{\Omega}^{+}$and $p^{-}=p_{\Omega}^{-}$. The variable exponent Lebesgue space $L_{p(\cdot)}(\Omega)$ consists of all measurable functions $f$ such that there exists an $\lambda>0$ such that the modular

$$
\varrho_{L_{p(\cdot)}(\Omega)}(f / \lambda)=\int_{\Omega} \varphi_{p(x)}\left(\frac{|f(x)|}{\lambda}\right) d x
$$

is finite, where

$$
\varphi_{p}(t)= \begin{cases}t^{p} & \text { if } p \in(0, \infty) \\ 0 & \text { if } p=\infty \text { and } t \leq 1 \\ \infty & \text { if } p=\infty \text { and } t>1\end{cases}
$$

Received by the editors February 1, 2011 and, in revised form, November 29, 2011.

2010 Mathematics Subject Classification. Primary 46E35.

Key words and phrases. Triangle inequality, Lebesgue spaces with variable exponent, iterated Lebesgue spaces.

The first author acknowledges the financial support provided by DFG project HA 2794/5-1, "Wavelets and Function Spaces on Domains".

The second author acknowledges the financial support provided by FWF project Y 432-N15 START-Preis, "Sparse Approximation and Optimization in High Dimensions".

The authors would like to thank the referee for useful hints, which helped to improve the paper. 
This definition is nowadays standard and was also used in [1, Section 2.2] and 3, Definition 3.2.1].

If we define $\Omega_{\infty}=\{x \in \Omega: p(x)=\infty\}$ and $\Omega_{0}=\Omega \backslash \Omega_{\infty}$, then the Luxemburg norm of a function $f \in L_{p(\cdot)}(\Omega)$ is given by

$$
\begin{aligned}
\left\|f \mid L_{p(\cdot)}(\Omega)\right\| & =\inf \left\{\lambda>0: \varrho_{L_{p(\cdot)}(\Omega)}(f / \lambda) \leq 1\right\} \\
& =\inf \left\{\lambda>0: \int_{\Omega_{0}}\left(\frac{|f(x)|}{\lambda}\right)^{p(x)} d x \leq 1 \text { and }|f(x)| \leq \lambda \text { for a.e. } x \in \Omega_{\infty}\right\} .
\end{aligned}
$$

If $p(\cdot) \geq 1$, then it is a norm, but it is always a quasi-norm if at least $p^{-}>0$; see 4 for details. We denote the class of all measurable functions $p: \mathbb{R}^{n} \rightarrow(0, \infty]$ such that $p^{-}>0$ by $\mathcal{P}\left(\mathbb{R}^{n}\right)$ and the corresponding modular is denoted by $\varrho_{p(\cdot)}$ instead

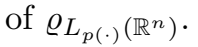

To define the mixed spaces $\ell_{q(\cdot)}\left(L_{p(\cdot)}\right)$ we have to define another modular. For $p, q \in \mathcal{P}\left(\mathbb{R}^{n}\right)$ and a sequence $\left(f_{\nu}\right)_{\nu \in \mathbb{N}_{0}}$ of $L_{p(\cdot)}\left(\mathbb{R}^{n}\right)$ functions we define

$$
\varrho_{\ell_{q(\cdot)}\left(L_{p(\cdot)}\right)}\left(f_{\nu}\right)=\sum_{\nu=0}^{\infty} \inf \left\{\lambda_{\nu}>0: \varrho_{p(\cdot)}\left(\frac{f_{\nu}}{\lambda_{\nu}^{1 / q(\cdot)}}\right) \leq 1\right\},
$$

where we put $\lambda^{1 / \infty}:=1$. The (quasi-) norm in the $\ell_{q(\cdot)}\left(L_{p(\cdot)}\right)$ spaces is defined as usual by

$$
\left\|f_{\nu} \mid \ell_{q(\cdot)}\left(L_{p(\cdot)}\right)\right\|=\inf \left\{\mu>0: \varrho_{\ell_{q(\cdot)}\left(L_{p(\cdot)}\right)}\left(f_{\nu} / \mu\right) \leq 1\right\} .
$$

This (quasi-)norm was used in 1 to define the spaces of Besov type with variable integrability and summability. Spaces of Triebel-Lizorkin type with variable indices have recently been considered in [2]. The appropriate $L_{p(\cdot)}\left(\ell_{q(\cdot)}\right)$ space is a normed space whenever ess-inf $x_{x \in \mathbb{R}^{n}} \min (p(x), q(x)) \geq 1$. This was the expected result and coincides with the case of constant exponents.

As pointed out in the remark after Theorem 3.8 in [1, the same question is still open for the $\ell_{q(\cdot)}\left(L_{p(\cdot)}\right)$ spaces.

\section{WhEn DOES $\left\|\cdot \mid \ell_{q(\cdot)}\left(L_{p(\cdot)}\right)\right\|$ DEFInE A NORM?}

In Theorem 3.6 of [1] the authors proved that if the condition $\frac{1}{p(x)}+\frac{1}{q(x)} \leq 1$ holds for almost every $x \in \mathbb{R}^{n}$, then $\left\|\cdot \mid \ell_{q(\cdot)}\left(L_{p(\cdot)}\right)\right\|$ defines a norm. They also proved in Theorem 3.8 that $\left\|\cdot \mid \ell_{q(\cdot)}\left(L_{p(\cdot)}\right)\right\|$ is a quasi-norm for all $p, q \in \mathcal{P}\left(\mathbb{R}^{n}\right)$. Furthermore, the authors of [1] posed a question if the (rather natural) condition $p(x), q(x) \geq 1$ for almost every $x \in \mathbb{R}^{n}$ ensures that $\left\|\cdot \mid \ell_{q(\cdot)}\left(L_{p(\cdot)}\right)\right\|$ is a norm.

We give (in Theorem 10 a positive answer if $1 \leq q(x) \leq p(x) \leq \infty$ almost everywhere on $\mathbb{R}^{n}$. Furthermore, in Theorem 2, we construct two functions $p(\cdot), q(\cdot) \in$ $\mathcal{P}\left(\mathbb{R}^{n}\right)$ such that $\inf _{x \in \mathbb{R}^{n}} \min (p(x), q(x)) \geq 1$, but the triangle inequality does not hold for $\left\|\cdot \mid \ell_{q(\cdot)}\left(L_{p(\cdot)}\right)\right\|$.

2.1. Positive results. We summarize in the following theorem all the cases when the expression $\left\|\cdot \mid \ell_{q(\cdot)}\left(L_{p(\cdot)}\right)\right\|$ is known to be a norm. We include the proof of the case discussed already in [1] for the sake of completeness.

Theorem 1. Let $p, q \in \mathcal{P}\left(\mathbb{R}^{n}\right)$ such that either $p(x) \geq 1$ and $q \geq 1$ is constant almost everywhere or $1 \leq q(x) \leq p(x) \leq \infty$ for almost every $x \in \mathbb{R}^{n}$, or $1 / p(x)+$ $1 / q(x) \leq 1$ for almost every $x \in \mathbb{R}^{n}$. Then $\left\|\cdot \mid \ell_{q(\cdot)}\left(L_{p(\cdot)}\right)\right\|$ defines a norm. 
Proof. If $p(x) \geq 1$ and $q \geq 1$ is constant almost everywhere, then the proof is trivial.

In the remaining cases, we want to show that

$$
\left\|f_{\nu}+g_{\nu}\left|\ell_{q(\cdot)}\left(L_{p(\cdot)}\right)\|\leq\| f_{\nu}\right| \ell_{q(\cdot)}\left(L_{p(\cdot)}\right)\right\|+\left\|g_{\nu} \mid \ell_{q(\cdot)}\left(L_{p(\cdot)}\right)\right\|
$$

for all sequences of measurable functions $\left\{f_{\nu}\right\}_{\nu \in \mathbb{N}_{0}}$ and $\left\{g_{\nu}\right\}_{\nu \in \mathbb{N}_{0}}$. Let $\mu_{1}>0$ and $\mu_{2}>0$ be given with

$$
\varrho_{\ell_{q(\cdot)}\left(L_{p(\cdot)}\right)}\left(\frac{f_{\nu}}{\mu_{1}}\right) \leq 1 \quad \text { and } \quad \varrho_{\ell_{q(\cdot)}\left(L_{p(\cdot)}\right)}\left(\frac{g_{\nu}}{\mu_{2}}\right) \leq 1 .
$$

We want to show that

$$
\varrho_{\ell_{q(\cdot)}\left(L_{p(\cdot)}\right)}\left(\frac{f_{\nu}+g_{\nu}}{\mu_{1}+\mu_{2}}\right) \leq 1 .
$$

For every $\varepsilon>0$, there exist sequences of positive numbers $\left\{\lambda_{\nu}\right\}_{\nu \in \mathbb{N}_{0}}$ and $\left\{\Lambda_{\nu}\right\}_{\nu \in \mathbb{N}_{0}}$ such that

$$
\varrho_{p(\cdot)}\left(\frac{f_{\nu}(x)}{\mu_{1} \lambda_{\nu}^{1 / q(x)}}\right) \leq 1 \quad \text { and } \quad \varrho_{p(\cdot)}\left(\frac{g_{\nu}(x)}{\mu_{2} \Lambda_{\nu}^{1 / q(x)}}\right) \leq 1
$$

together with

$$
\sum_{\nu=0}^{\infty} \lambda_{\nu} \leq 1+\varepsilon \quad \text { and } \quad \sum_{\nu=0}^{\infty} \Lambda_{\nu} \leq 1+\varepsilon
$$

We set

$$
A_{\nu}:=\frac{\mu_{1} \lambda_{\nu}+\mu_{2} \Lambda_{\nu}}{\mu_{1}+\mu_{2}}, \text { i.e. } \sum_{\nu=0}^{\infty} A_{\nu} \leq 1+\varepsilon .
$$

We shall prove that

$$
\varrho_{p(\cdot)}\left(\frac{f_{\nu}(x)+g_{\nu}(x)}{A_{\nu}^{1 / q(x)}\left(\mu_{1}+\mu_{2}\right)}\right) \leq 1 \quad \text { for all } \quad \nu \in \mathbb{N}_{0} .
$$

Let $\Omega_{0}:=\left\{x \in \mathbb{R}^{n}: p(x)<\infty\right\}$ and $\Omega_{\infty}:=\left\{x \in \mathbb{R}^{n}: p(x)=\infty\right\}$. We put for every $x \in \Omega_{0}$

$$
F_{\nu}(x):=\left(\frac{\left|f_{\nu}(x)\right|}{\mu_{1} \lambda_{\nu}^{1 / q(x)}}\right)^{p(x)} \quad \text { and } \quad G_{\nu}(x):=\left(\frac{\left|g_{\nu}(x)\right|}{\mu_{2} \Lambda_{\nu}^{1 / q(x)}}\right)^{p(x)} .
$$

Then (11) may be reformulated as

$$
\int_{\Omega_{0}} F_{\nu}(x) d x \leq 1 \quad \text { and } \quad \operatorname{ess-sup}_{x \in \Omega_{\infty}} \frac{\left|f_{\nu}(x)\right|}{\mu_{1} \lambda_{\nu}^{1 / q(x)}} \leq 1
$$

and

$$
\int_{\Omega_{0}} G_{\nu}(x) d x \leq 1 \quad \text { and } \quad \operatorname{ess}_{x \in \Omega_{\infty}} \frac{\left|g_{\nu}(x)\right|}{\mu_{2} \Lambda_{\nu}^{1 / q(x)}} \leq 1 .
$$

Our aim is to prove (2), which reads

$$
\int_{\Omega_{0}}\left(\frac{\left|f_{\nu}(x)+g_{\nu}(x)\right|}{A_{\nu}^{1 / q(x)}\left(\mu_{1}+\mu_{2}\right)}\right)^{p(x)} d x \leq 1 \quad \text { and } \quad \operatorname{ess-sup}_{x \in \Omega_{\infty}} \frac{\left|f_{\nu}(x)+g_{\nu}(x)\right|}{A_{\nu}^{1 / q(x)}\left(\mu_{1}+\mu_{2}\right)} \leq 1 .
$$


We first prove the second part of (5). First we observe that (3) and (4) imply that

$$
\left|f_{\nu}(x)\right| \leq \mu_{1} \lambda_{\nu}^{1 / q(x)} \quad \text { and } \quad\left|g_{\nu}(x)\right| \leq \mu_{2} \Lambda_{\nu}^{1 / q(x)}
$$

hold for almost every $x \in \Omega_{\infty}$. Using $q(x) \geq 1$ and Hölder's inequality in the form

$$
\frac{\mu_{1} \lambda_{\nu}^{1 / q(x)}+\mu_{2} \Lambda_{\nu}^{1 / q(x)}}{\mu_{1}+\mu_{2}} \leq\left(\frac{\mu_{1} \lambda_{\nu}+\mu_{2} \Lambda_{\nu}}{\mu_{1}+\mu_{2}}\right)^{1 / q(x)},
$$

we get

$$
\frac{\left|f_{\nu}(x)+g_{\nu}(x)\right|}{A_{\nu}^{1 / q(x)}\left(\mu_{1}+\mu_{2}\right)} \leq 1 .
$$

If $q(x)=\infty$, only notational changes are necessary.

Next we prove the first part of (5). Let $1 \leq q(x) \leq p(x)<\infty$ for almost all $x \in \Omega_{0}$. Then we use Hölder's inequality in the form

(6)

$$
\begin{aligned}
& F_{\nu}(x)^{1 / p(x)} \lambda_{\nu}^{1 / q(x)} \mu_{1}+G_{\nu}(x)^{1 / p(x)} \Lambda_{\nu}^{1 / q(x)} \mu_{2} \\
& \quad \leq\left(\mu_{1}+\mu_{2}\right)^{1-1 / q(x)}\left(\mu_{1} \lambda_{\nu}+\mu_{2} \Lambda_{\nu}\right)^{1 / q(x)-1 / p(x)}\left(F_{\nu}(x) \lambda_{\nu} \mu_{1}+G_{\nu}(x) \Lambda_{\nu} \mu_{2}\right)^{1 / p(x)} .
\end{aligned}
$$

If $1 / p(x)+1 / q(x) \leq 1$ for almost every $x \in \Omega_{0}$, then we replace (6) by

(7) $F_{\nu}(x)^{1 / p(x)} \lambda_{\nu}^{1 / q(x)} \mu_{1}+G_{\nu}(x)^{1 / p(x)} \Lambda_{\nu}^{1 / q(x)} \mu_{2}$

$$
\leq\left(\mu_{1}+\mu_{2}\right)^{1-1 / p(x)-1 / q(x)}\left(\mu_{1} \lambda_{\nu}+\mu_{2} \Lambda_{\nu}\right)^{1 / q(x)}\left(F_{\nu}(x) \mu_{1}+G_{\nu}(x) \mu_{2}\right)^{1 / p(x)} .
$$

Using (6), we may continue:

$$
\begin{aligned}
& \int_{\Omega_{0}}\left(\frac{\left|f_{\nu}(x)+g_{\nu}(x)\right|}{A_{\nu}^{1 / q(x)}\left(\mu_{1}+\mu_{2}\right)}\right)^{p(x)} d x \\
& =\int_{\Omega_{0}}\left(\frac{F_{\nu}(x)^{1 / p(x)} \lambda_{\nu}^{1 / q(x)} \mu_{1}+G_{\nu}(x)^{1 / p(x)} \Lambda_{\nu}^{1 / q(x)} \mu_{2}}{\mu_{1}+\mu_{2}}\right)^{p(x)} \cdot\left(\frac{\mu_{1} \lambda_{\nu}+\mu_{2} \Lambda_{\nu}}{\mu_{1}+\mu_{2}}\right)^{-\frac{p(x)}{q(x)}} d x \\
& \leq \int_{\Omega_{0}} \frac{F_{\nu}(x) \lambda_{\nu} \mu_{1}+G_{\nu}(x) \Lambda_{\nu} \mu_{2}}{\mu_{1} \lambda_{\nu}+\mu_{2} \Lambda_{\nu}} d x \\
& =\frac{\mu_{1} \lambda_{\nu}}{\mu_{1} \lambda_{\nu}+\mu_{2} \Lambda_{\nu}} \int_{\Omega_{0}} F_{\nu}(x) d x+\frac{\mu_{2} \Lambda_{\nu}}{\mu_{1} \lambda_{\nu}+\mu_{2} \Lambda_{\nu}} \int_{\Omega_{0}} G_{\nu}(x) d x \leq 1,
\end{aligned}
$$

where we also used (3) and (4). If we start with (7) instead, we proceed in the following way:

$$
\begin{aligned}
& \int_{\Omega_{0}}\left(\frac{\left|f_{\nu}(x)+g_{\nu}(x)\right|}{A_{\nu}^{1 / q(x)}\left(\mu_{1}+\mu_{2}\right)}\right)^{p(x)} d x \\
& =\int_{\Omega_{0}}\left(\frac{F_{\nu}(x)^{1 / p(x)} \lambda_{\nu}^{1 / q(x)} \mu_{1}+G_{\nu}(x)^{1 / p(x)} \Lambda_{\nu}^{1 / q(x)} \mu_{2}}{\mu_{1}+\mu_{2}}\right)^{p(x)} \cdot\left(\frac{\mu_{1} \lambda_{\nu}+\mu_{2} \Lambda_{\nu}}{\mu_{1}+\mu_{2}}\right)^{-\frac{p(x)}{q(x)}} d x \\
& \leq \int_{\Omega_{0}} \frac{F_{\nu}(x) \mu_{1}+G_{\nu}(x) \mu_{2}}{\mu_{1}+\mu_{2}} d x=\frac{\mu_{1}}{\mu_{1}+\mu_{2}} \int_{\Omega_{0}} F_{\nu}(x) d x+\frac{\mu_{2}}{\mu_{1}+\mu_{2}} \int_{\Omega_{0}} G_{\nu}(x) d x \leq 1 .
\end{aligned}
$$

In both cases, this finishes the proof of (5). 
Remark 1. (i) A simpler proof of Theorem 1 is possible (and was proposed to us by the referee) if $1 \leq q(x) \leq p(x) \leq \infty$. Namely, if $1 \leq q \leq p \leq \infty, \lambda>0$ and $t \geq 0$, then

$$
\varphi_{p}\left(\frac{t}{\lambda^{1 / q}}\right)=\varphi_{\frac{p}{q}}\left(\frac{\varphi_{q}(t)}{\lambda}\right)
$$

where we use the convention that $\frac{p}{q}=1$ if $p=q=\infty$. This allows us to simplify the modular $\varrho_{\ell_{q(\cdot)}\left(L_{p(\cdot)}\right)}$ to

$$
\varrho_{\ell_{q(\cdot)}\left(L_{p(\cdot)}\right)}\left(f_{\nu}\right)=\sum_{\nu=0}^{\infty}\left\|\varphi_{q(\cdot)}\left(\left|f_{\nu}\right|\right)\right\|_{\frac{p(\cdot)}{q(\cdot)}} .
$$

This shows that $\varrho_{\ell_{q(\cdot)}\left(L_{p(\cdot)}\right)}\left(f_{\nu}\right)$ is a composition of only convex functions. Hence, it is a convex modular, and therefore it induces a norm. Unfortunately, we were not able to find such a simplification for the case $1 / p(x)+1 / q(x) \leq 1$. The advantage of our proof of Theorem 1 is that it proves both cases in a unified way.

(ii) Let us observe that (8) loses its sense if $p<q=\infty$. This shows why (9) (which was already used in [1] for $q^{+}<\infty$ ) has to be applied with certain care.

(iii) The method of the proof of Theorem 1 can actually be used to show that under the conditions posed on $p(\cdot)$ and $q(\cdot)$ in Theorem $1, \varrho_{\ell_{q(\cdot)}\left(L_{p(\cdot)}\right)}$ is a convex modular, which is a stronger result than the norm property.

\subsection{Counterexample.}

Theorem 2. There exist functions $p, q \in \mathcal{P}\left(\mathbb{R}^{n}\right)$ with $\inf _{x \in \mathbb{R}^{n}} p(x) \geq 1$ and $\inf _{x \in \mathbb{R}^{n}} q(x) \geq 1$ such that $\| \cdot\left|\ell_{q(\cdot)}\left(L_{p(\cdot)}\right)\right| \mid$ does not satisfy the triangle inequality.

Proof. Let $Q_{0}, Q_{1} \subset \mathbb{R}^{n}$ be two disjoint unit cubes, let $p(x):=1$ everywhere on $\mathbb{R}^{n}$, and put $q(x):=\infty$ for $x \in Q_{1}$ and $q(x):=1$ for $x \notin Q_{1}$. Let $f_{1}=\chi_{Q_{0}}$ and $f_{2}=\chi_{Q_{1}}$. Finally, we put $f=\left(f_{1}, f_{2}, 0, \ldots\right)$ and $g=\left(f_{2}, f_{1}, 0, \ldots\right)$.

We calculate for every $L>0$ fixed

$$
\inf \left\{\lambda_{1}>0: \varrho_{p(\cdot)}\left(\frac{f_{1}(x)}{\lambda_{1}^{1 / q(x)} L}\right) \leq 1\right\}=\inf \left\{\lambda_{1}>0: \frac{1}{\lambda_{1} L} \leq 1\right\}=1 / L
$$

and

$$
\inf \left\{\lambda_{2}>0: \varrho_{p(\cdot)}\left(\frac{f_{2}(x)}{\lambda_{2}^{1 / q(x)} L}\right) \leq 1\right\}=\inf \left\{\lambda_{2}>0: \frac{1}{L} \leq 1\right\} .
$$

If $L \geq 1$, then the last expression is equal to zero; otherwise it is equal to $\infty$.

We obtain

$\left\|f \mid \ell_{q(\cdot)}\left(L_{p(\cdot)}\right)\right\|=\inf \left\{L>0: \varrho_{\ell_{q(\cdot)}\left(L_{p(\cdot)}\right)}(f / L) \leq 1\right\}=\inf \{L>0: 1 / L+0 \leq 1\}=1$,

and the same is also true for $\left\|g \mid \ell_{q(\cdot)}\left(L_{p(\cdot)}\right)\right\|$. It is therefore enough to show that $\left\|f+g \mid \ell_{q(\cdot)}\left(L_{p(\cdot)}\right)\right\|>2$. 
Using the calculation

$$
\begin{aligned}
\inf \{\lambda & \left.>0: \varrho_{p(\cdot)}\left(\frac{f_{1}(x)+f_{2}(x)}{L \cdot \lambda^{1 / q(x)}}\right) \leq 1\right\}=\inf \left\{\lambda>0: \int_{Q_{0}} \frac{1}{L \cdot \lambda}+\int_{Q_{1}} \frac{1}{L} \leq 1\right\} \\
& =\inf \left\{\lambda>0: \frac{1}{L \cdot \lambda}+\frac{1}{L} \leq 1\right\}=\frac{1}{L-1},
\end{aligned}
$$

which holds for every $L>1$ fixed, we get

$$
\begin{aligned}
\left\|f+g \mid \ell_{q(\cdot)}\left(L_{p(\cdot)}\right)\right\| & =\inf \left\{L>0: \varrho_{\ell_{q(\cdot)}\left(L_{p(\cdot)}\right)}\left(\frac{f+g}{L}\right) \leq 1\right\} \\
& =\inf \left\{L>0: 2 \inf \left\{\lambda>0: \varrho_{p(\cdot)}\left(\frac{f_{1}(x)+f_{2}(x)}{L \cdot \lambda^{1 / q(x)}}\right) \leq 1\right\} \leq 1\right\} \\
& =\inf \left\{L>1: 2 \cdot \frac{1}{L-1} \leq 1\right\}=3 .
\end{aligned}
$$

Remark 2. Let us observe that $1 \leq q(x) \leq p(x) \leq \infty$ holds for $x \in Q_{0}$ and that $1 / p(x)+1 / q(x) \leq 1$ is true for $x \in Q_{1}$. It is therefore necessary to interpret the assumptions of Theorem 1 in a correct way, namely, that one of the conditions of Theorem 1 holds for (almost) all $x \in \mathbb{R}^{n}$. This is not to be confused with the statement that for (almost) every $x \in \mathbb{R}^{n}$ at least one of the conditions is satisfied, which is not sufficient.

Remark 3. A similar calculation (which we shall not repeat in detail) shows that one may also put $q(x):=q_{0}$ large enough for $x \in Q_{1}$ to obtain a counterexample. Hence there is nothing special about the infinite value of $q$, and the same counterexample may be reproduced with uniformly bounded exponents $p, q \in \mathcal{P}\left(\mathbb{R}^{n}\right)$.

\section{References}

1. A. Almeida, P. Hästö: Besov spaces with variable smoothness and integrability, J. Funct. Anal. (5) 258 (2010), 1628-1655. MR2566313(2011e:46045)

2. L. Diening, P. Hästö, S. Roudenko: Function spaces of variable smoothness and integrability, J. Funct. Anal. (6) 256 (2009), 1731-1768. MR2498558 (2010b:46067)

3. L. Diening, P. Harjulehto, P. Hästö, M. Růžička: Lebesgue and Sobolev Spaces with Variable Exponents, Springer Lecture Notes in Mathematics 2017 (2011). MR2790542

4. O. Kováčik, J. Rákosník: On spaces $L^{p(x)}$ and $W^{1, p(x)}$, Czechoslovak Math. J. 41 (116) (1991), 592-618. MR1134951 (92m:46047)

5. W. Orlicz: Über konjugierte Exponentenfolgen, Studia Math. 3 (1931), 200-212.

Mathematical Institute, Friedrich-Schiller-University, D-07737 Jena, Germany

E-mail address: henning.kempka@uni-jena.de

RiCAM, Austrian Academy of Sciences, Altenbergstrasse 69, A-4040 Linz, Austria

E-mail address: jan.vybiral@oeaw.ac.at 